\title{
To Stent or Not to Stent? This is the Renal Artery Stenosis Question
}

\author{
Michael Doumas ${ }^{1,2}$, Konstantinos Tziomalos $^{3}$ and Vasilios G. Athyros, ${ }^{1, *}$
}

\begin{abstract}
${ }^{I}$ Second Prop. Department of Internal Medicine, Medical School, Aristotle University of Thessaloniki, Hippokration Hospital, Thessaloniki, Greece; ${ }^{2}$ Veteran Administration Medical Center and George Washington University, Washington, DC, USA; ${ }^{3}$ First Prop. Department of Internal Medicine, Medical School, Aristotle University of Thessaloniki, AHEPA Hospital, Thessaloniki, Greece
\end{abstract}

Keywords: Renal artery stenosis, renal artery stenting, antihypertensive drug treatment, cardiovascular events, superiority.

A few days ago (Nov 18, 2013) the results of the large prospective Cardiovascular Outcomes in Renal Atherosclerotic Lesions (CORAL) trial were presented and published. CORAL enrolled 947 participants who had atherosclerotic renal artery stenosis (ARAS), $\geq 60 \%$ and either uncontrolled systolic hypertension despite treatment with $\geq 2$ anti-hypertensive drugs or stage 3 chronic kidney disease (CKD) [1]. CORAL randomly assigned patients to medical therapy plus renal-artery stenting or medical therapy alone. The median follow-up period was 43 months and the primary endpoint was a composite of death from cardiovascular disease (CVD) or renal causes, myocardial infarction (MI), stroke, congestive heart failure, progression of renal insufficiency, or need for renal replacement therapy. Results showed that the addition of renal-artery stenting to thorough multifactorial medical therapy did not contribute a significant clinical benefit with respect to the prevention of CVD events or renal function deterioration. Adverse CVD or renal events occurred in $35.1 \%$ of patients who received stenting and $35.8 \%$ of those on medical therapy alone $(p=0.58)$ [1]. Thus, the authors concluded that renal artery stenting was not superior to optimal medical therapy alone for moderately severe ARAS [1].

The results of CORAL have a direct impact on clinical practice questioning the need for renal artery stenting in patients managed with optimal medical therapy. The message seems to be very simple. If patients have ARAS and high blood pressure, they need a very aggressive comprehensive medical therapy before considering any interventional procedure.

A recent meta-analysis investigated CVD outcome in patients with ARAS treated either with renal angioplasty or antihypertensive drug therapy [2]. Pooled data from 5 studies $(\mathrm{n}=1,159$ patients) showed that during the 2-year follow-up there were no significant clinical outcome differences between angioplasty and medical therapy [2], both in patients

\footnotetext{
*Address correspondence to this author at the Head of Metabolic Disease Units, 2nd Prop. Department of Internal Medicine, Medical School, Aristotle University of Thessaloniki, Hippokration Hospital, Thessaloniki, Greece. 15 Marmara St, Thessaloniki, 55132, Greece; Tel: +30 2310 892606; Fax: +30 2310 835955;

E-mails: athyros@med.auth.gr or vathyros@gmail.com
}

with or without CKD. In another study [3] it was shown that patients with ARAS and normal or near normal renal function (creatinine $\leq 2.0 \mathrm{mg} / \mathrm{dl}$ ) can be safely treated with effective drug treatment, with a small decrease in GFR. For patients who have atherosclerotic MI, stroke or renal impairment, renal artery stenting may further reduce blood pressure and delay the deterioration or even improve renal function [3].

The Angioplasty and Stenting for Renal Artery Lesions (ASTRAL) trial $(\mathrm{n}=867)$ [4], although criticised by some for methodological issues [5], results showed that there were no meaningful clinical benefit from revascularization in patients with ARAS; however considerable risks were present [4].

On the other hand, in patients with renovascular hypertension due to fibromuscular dysplasia (RAFMD) and/or the combination of RAFMD with ARAS, angioplasty is the method of choice and has favourable short- and long-term CVD and renal clinical outcomes [6].

The take away message from all the above is that prior trials showed similar results in mild ARAS; this study established the futility of stenting for a higher severity (moderate severity) of the disease. Substantially severe forms of ARAS will probably have to undergo renal angioplasty with stenting, especially when flash pulmonary oedema occurs [7-9].

\section{CONFLICT OF INTEREST}

The author(s) confirm that this article content has no conflicts of interest.

\section{ACKNOWLEDGEMENT}

Declared none.

\section{REFERENCES}

[1] Cooper CJ, Murphy TP, Cutlip DE, et al.; for the CORAL Investigators. Stenting and Medical Therapy for Atherosclerotic RenalArtery Stenosis. N Engl J Med 2013 Nov 18. [Epub ahead of print]

[2] Pierdomenico SD, Pierdomenico AM, Cuccurullo C, Mancini M, Di Carlo S, Cuccurullo F. Cardiac events in hypertensive patients with renal artery stenosis treated with renal angioplasty or drug therapy: meta-analysis of randomized trials. Am J Hypertens 2012; 25: 1209-14.

[3] Hanzel G, Balon H, Wong O, Soffer D, Lee DT, Safian RD. Prospective evaluation of aggressive medical therapy for atheroscle- 
rotic renal artery stenosis, with renal artery stenting reserved for previously injured heart, brain, or kidney. Am J Cardiol 2005; 96: 1322-27.

[4] ASTRAL Investigators, Wheatley K, Ives N, Gray R, Revascularization versus medical therapy for renal-artery stenosis. N Engl $\mathrm{J}$ Med 2009; 361(20): 1953-62.

[5] George JC, White CJ. Renal artery stenting: lessons from ASTRAL (Angioplasty and Stenting for Renal Artery Lesions). JACC Cardiovasc Interv 2010; 3: 786-7.

[6] Mousa AY, Campbell JE, Stone PA, Broce M, Bates MC, AbuRahma AF. Short- and long-term outcomes of percutaneous transluminal angioplasty/stenting of renal fibromuscular dysplasia over a ten-year period. J Vasc Surg 2012; 55: 421-7.

[7] Stavrinou E, Koumaras C, Gossios TD, Tziomalos K, Athyros VG, Karagiannis A. Atherosclerotic renal artery stenosis: an update on diagnosis and management. Curr Vasc Pharmacol 2011; 9: 465-70.

[8] Karagiannis A, Tziomalos K, Anagnostis P, Gossios T, Athyros VG. Atherosclerotic renal artery stenosis: medical therapy alone or in combination with revascularization? Angiology 2009; 60: 397402.

[9] Faselis C, Doumas M, Papademetriou V. Common secondary causes of resistant hypertension and rational for treatment. Int J Hypertens 2011; 2011: 236-9.

(C) Doumas et al.; Licensee Bentham Open.

This is an open access article licensed under the terms of the Creative Commons Attribution Non-Commercial License (http://creativecommons.org/licenses/by-nc/3.0/) which permits unrestricted, non-commercial use, distribution and reproduction in any medium, provided the work is properly cited. 